\title{
$p$-FACTORABLE OPERATORS
}

\author{
BY \\ C. V. HUTTON( $\left.{ }^{1}\right)$
}

\begin{abstract}
Several classes of operators on Banach spaces, defined by certain summability conditions on the $k$ th approximation numbers, are introduced and studied. Characterizations of these operators in terms of tensor-product representations are obtained. The relationship between these operators and other classes of operators introduced by various authors is studied in some detail.
\end{abstract}

Introduction. This paper is a study of a class of operators on Banach spaces which we call the $p$-factorable operators. The notion of a $p$-factorable operator is a natural extension to the operators of type $l^{p}$ of ideals of Grothendieck concerning the factorization of nuclear operators. Since it is easier to study properties of diagonal operators on the $l^{p}$ spaces, it is useful to know which operators admit such factorizations. We give a characterization of the $p$-factorable operators in terms of certain tensor product representations. Such representations are useful, for example, when studying the summability properties of the eigenvalues of an operator. The classical theorem of $\mathrm{H}$. Weyl discussed in $\S 1$ is an example of this. In $\$ 2$ we study in some detail the relationship between the $p$-factorable operators and several other classes of operators on Banach spaces which have been introduced by several authors.

We begin by giving basic definitions and establish the notation which will be used throughout this paper.

All spaces considered are Banach spaces. We denote the unit ball of $E$ by $U_{E}$ and the space of all continuous linear functionals on $E$ by $E^{\prime}$. By operator we mean a bounded linear transformation. The collection of all operators from $E$ to $F$ will be denoted by $L(E, F)$ and $\|T\|$ denotes the usual operator norm for $T \in L(E, F)$. For $T \in L(E, F)$ the astriction of $T, T_{a}$, is the operator $T_{a}: E \rightarrow$ $\overline{T(E)}$ defined by $T_{a} x=T x$ for all $x \in E$. If $f \in E^{\prime}$ and $y \in F$ then by $f \otimes y$ we mean the rank one operator $f \otimes y: E \rightarrow F$ defined by $f \otimes y(x)=$ $\langle x, f\rangle y$ for every $x \in E$.

We will be interested in the following classes of operators:

The operators of type $l^{p}$. For $T \in L(E, F)$ the kth approximation number of $T, \alpha_{k}(T)$, is defined by $\alpha_{k}(T)=\inf \|T-A\|$, the inf being taken over all

Received by the editors December 19, 1973.

AMS(MOS) subject classifications (1970). Primary 41A45, 47B99; Secondary 47B05.

(1)Portions of this paper appear in the dissertation of the author written at Louisiana State University under the direction of J. R. Retherford. 
$A \in L(E, F)$ of rank at most $k$. For detailed information concerning properties of the $k$ th approximation numbers and their relationship to other approximation numbers associated with operators on Banach spaces see [2], [5], [6], [7] and [8].

Following Pietsch we say an operator $T \in L(E, F)$ is of type $l^{p}, 0<$ $p \leqslant \infty$, if the sequence $\left(\alpha_{k}(T)\right)_{k=0}^{\infty}$ belongs to $l^{p}$ (or to $c_{0}$ if $p=\infty$ ). We denote the collection of operators of type $l^{p}$ from $E$ to $F$ by $l^{p}(E, F)$.

The strongly p-summable operators. In the second chapter of his memoir, Grothendieck [1] introduced a class of operators on Banach spaces which he called operators "de puissance $p$. ème sommable" for $0<p \leqslant 1$. To avoid confusion with other classes of operators we shall refer to these operators as strongly $p$-summable. Namely $T \in L(E, F)$ is said to be strongly p-summable, $0<p \leqslant$ 1, if $T$ has a representation $T=\sum_{i=1}^{\infty} \lambda_{i} f_{i} \otimes y_{i}$ where $\left(\lambda_{i}\right)_{i=1}^{\infty} \in l^{p},\left(f_{i}\right)_{i=1}^{\infty} \subset$ $U_{E^{\prime}}$, and $\left(y_{i}\right)_{i=1}^{\infty} \subset U_{F}$. Following Grothendieck we will let $L^{(p)}(E, F)$ denote the complete, metrizable, topological vector space of all strongly $p$-summable operators from $E$ to $F$ given the topology generated by

$$
S_{p}(T)=\inf \left\{\sum_{i=1}^{\infty}\left|\lambda_{i}\right|^{p}: T=\sum_{i=1}^{\infty} \lambda_{i} f_{i} \otimes y_{i}\right\} .
$$

The operators of Markus. The class of operators $F_{p}(E, F), 0<p \leqslant 1$, was introduced and studied by Markus in [4] and is defined to be the collection of all $T \in L(E, F)$ having a representation $T=\sum_{i=1}^{\infty} \lambda_{i} f_{i} \otimes y_{i}$ with $\left(f_{i}\right)_{i=1}^{\infty} \subset U_{E^{\prime}}$, $\left(y_{i}\right)_{i=1}^{\infty} \subset U_{F}$ and $\left(\lambda_{i}\right)_{i=1}^{\infty}=o\left(i^{-1 / p}\right)$. Given the topology generated by

$$
F_{p}(T)=\inf \left\{\sup _{n} n^{1 / p}\left|\lambda_{n}\right|: T=\sum_{n=1}^{\infty} \lambda_{n} f_{n} \otimes y_{n}\right\},
$$

$F_{p}(E, F)$ is complete and metrizable. For results pertaining to the relation between $F_{p}(E, F)$ and various other classes of operators see [4].

1. $p$-factorable operators. In his classic memoir [1], Grothendieck showed that a nuclear operator on arbitrary Banach spaces factors through a nuclear diagonal operator from $l^{\infty}$ to $l^{1}$ (by a diagonal operator $T \sim\left(\lambda_{i}\right)_{i=1}^{\infty}$ between sequence spaces we mean the operator $T\left(\xi_{i}\right)_{i=1}^{\infty}=\left(\lambda_{i} \xi_{i}\right)_{i=1}^{\infty}$ where the $\lambda_{i}$ are scalars). Since every operator of type $l^{1}$ is nuclear [6], the question naturally arises whether operators of type $l^{1}$ factor in a similar manner through a type $l^{1}$ diagonal. As we shall see this is not the case. Indeed, several new spaces of operators arise as natural extensions of the nuclear factorization result of Grothendieck. Moreover, these operators can be completely characterized by certain tensor product representations.

We first give a characterization of the diagonal operators from $l^{\infty}$ to $l^{1}$ 
which are of type $l^{1}$. Without loss of generality we may assume that for diagonals $T \sim\left(\lambda_{i}\right)_{i=1}^{\infty}$ we have $\left|\lambda_{i}\right| \geqslant\left|\lambda_{i+1}\right|$ for all $i$ (see, e.g., [2], [6]).

Proposition 1.1. If $T: l^{\infty} \rightarrow l^{1}$ is a diagonal, $T \sim\left(\lambda_{i}\right)_{i=1}^{\infty}$, then $\alpha_{k}(T)=$ $\sum_{i=k+1}^{\infty}\left|\lambda_{i}\right|$ for each $k$.

A proof of (1.1) can be found in [2]. The next result now follows immediately.

ThEOREM 1.2. A diagonal $T: l^{\infty} \rightarrow l^{1}$ with $T \sim\left(\lambda_{i}\right)_{i=1}^{\infty}$ is of type $l^{1}$ if and only if $\Sigma_{i=1}^{\infty} i\left|\lambda_{i}\right|$ converges.

It follows that if $T \in L(E, F)$ has a representation $T=\sum_{i=1}^{\infty} \lambda_{i} f_{i} \otimes y_{i}$ where $\left(f_{i}\right)_{i=1}^{\infty} \subset U_{E^{\prime}},\left(y_{i}\right)_{i=1}^{\infty} \subset U_{F}$ and $\Sigma_{i=1}^{\infty} i\left|\lambda_{i}\right|$ is finite then $T$ factors through a diagonal from $l^{\infty}$ to $l^{1}$ which is of type $l^{1}$. Indeed, we have

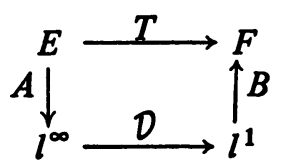

where $A x=\left(\left(x, f_{i}\right)\right)_{i=1}^{\infty}, D \sim\left(\lambda_{i}\right)_{i=1}^{\infty}$ and $B\left(\xi_{i}\right)_{i=1}^{\infty}=\sum_{i=1}^{\infty} \xi_{i} y_{i}$. Since $\alpha_{k}(D) \leqslant$ $\sum_{i=k+1}^{\infty}\left|\lambda_{i}\right|$ we have $\sum_{k=1}^{\infty} \alpha_{k-1}(D) \leqslant \sum_{k=1}^{\infty} \Sigma_{i=k}^{\infty}\left|\lambda_{i}\right| \leqslant \Sigma_{i=1}^{\infty} i\left|\lambda_{i}\right|$; hence $D$ is of type $l^{1}$.

We next show that this representation actually characterizes those operators which factor through a diagonal of type $l^{1}$ from $l^{\infty}$ to $l^{1}$.

Definition 1.3. We will say that $T \in L(E, F)$ is $l^{1}$-factorable if $T$ factors through a diagonal of type $l^{1}$ from $l^{\infty}$ to $l^{1}$.

Proposition 1.4. An operator $T \in L(E, F)$ is $l^{1}$-factorable if and only if $T$ has a representation $T=\sum_{i=1}^{\infty} \lambda_{i} f_{i} \otimes y_{i}$ where $\left(f_{i}\right)_{i=1}^{\infty} \subset U_{E^{\prime}},\left(y_{i}\right)_{i=1}^{\infty} \subset$ $U_{F}$ and $\sum_{i=1}^{\infty} i\left|\lambda_{i}\right|$ converges.

Proof. We have already observed the necessity, so suppose that $T$ has such a factorization. Then $T=B D A$ where

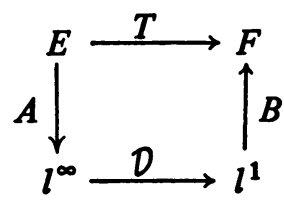

with $D \sim\left(\lambda_{i}\right)_{i=1}^{\infty}$. We may assume that $\left|\lambda_{i}\right| \geqslant\left|\lambda_{i+1}\right|$ for all $i$ by a permutation of the indices if necessary. Since $D \in l^{1}\left(l^{\infty}, l^{1}\right)$ it follows from (1.2) that $\sum_{i=1}^{\infty} i\left|\lambda_{i}\right|$ converges. Define $f_{i} \in E^{\prime}$ by $f_{i} x=(1 /\|A\|)\left\langle e_{i}, A x\right\rangle$ where $e_{i}$ is the $i$ th unit vector and let $y_{i}=(1 /\|B\|) B e_{i}$. If $\mu_{i}=\lambda_{i}\|A\|\|B\|$ then for $x \in E$ we 
have

$$
\begin{aligned}
\sum_{i=1}^{\infty} \mu_{i}\left\langle x, f_{i}\right\rangle y_{i} & =\sum_{i=1}^{\infty} \lambda_{i}\left\langle e_{i}, A x\right\rangle B e_{i}=B\left(\sum_{i=1}^{\infty} \lambda_{i}\left\langle e_{i}, A x\right\rangle e_{i}\right) \\
& =B D\left(\left\langle e_{i}, A x\right\rangle\right)_{i=1}^{\infty}=B D A x=T x ;
\end{aligned}
$$

hence $T=\sum_{i=1}^{\infty} \mu_{i} f_{i} \otimes y_{i}$ is the desired representation.

Clearly $l^{1}$-factorable operators are of type $l^{1}$. However, the two classes of operators are, in general, distinct. To see this we need the following theorem of H. Weyl [10] (see also $[1, \S 2]$ ).

THEOREM 1.5. Let $H$ be a Hilbert space and $0<p \leqslant 1$. If $T \in L^{(p)}(H, H)$ and $\left(z_{i}\right)_{i=1}^{\infty}$ is the sequence of eigenvalues of $T$, arranged in order of decreasing modulus and repeated according to multiplicity, then $\dot{\Sigma}_{i=1}^{\infty}\left|z_{i}\right|^{p} \leqslant S_{p}(T)$.

Now let $T: l^{2} \rightarrow l^{2}$ be the diagonal $T \sim\left(i^{-3 / 2}\right)_{i=1}^{\infty}$. That $T$ is of type $l^{1}$ follows from [6], [7]. If $T$ were $l^{1}$-factorable then by (1.4) there exist $\left(f_{i}\right)_{i=1}^{\infty} \subset U_{l^{2}},\left(y_{i}\right)_{i=1}^{\infty} \subset U_{l^{2}}$ and scalars $\left(\mu_{i}\right)_{i=1}^{\infty}$ with $\sum_{i=1}^{\infty} i\left|\mu_{i}\right|$ finite such that $T=\Sigma_{i=1}^{\infty} \mu_{i} f_{i} \otimes y_{i}$. But then

$$
\sum_{i=1}^{\infty}\left|\mu_{i}\right|^{2 / 3}=\sum_{i=1}^{\infty}\left(i\left|\mu_{i}\right|\right)^{2 / 3} i^{-2 / 3} \leqslant\left(\sum_{i=1}^{\infty} i\left|\mu_{i}\right|\right)^{2 / 3}\left(\sum_{i=1}^{\infty} i^{-2}\right)^{1 / 3}<+\infty
$$

hence $T \in L^{(2 / 3)}\left(l^{2}, l^{2}\right)$. That is, $S_{2 / 3}(T)$ is finite. But since the sequence of eigenvalues of $T$ is precisely $\left(i^{-3 / 2}\right)_{i=1}^{\infty}$ it follows from $(1.5)$ that $S_{2 / 3}(T) \geqslant$ $\sum_{i=1}^{\infty}\left(i^{-3 / 2}\right)^{2 / 3}=\sum_{i=1}^{\infty} i^{-1}$. This contradiction implies that $T$ is not $l^{1}$-factorable.

Definition 1.6. We will say that $T \in L(E, F)$ is $p$-factorable, $p \geqslant 1$, if $T$ factors through a diagonal $D: l^{\infty} \rightarrow l^{1}$ having the property that $\sum_{n=1}^{\infty} n^{p-1} \alpha_{n-1}(D)$ converges. We will denote the collection of $p$-factorable operators from $E$ to $F$ by $F_{p}(E, F)$.

It is clear that $F_{1}(E, F)$ coincides with the $l^{1}$-factorable operators from $E$ to $F$. In fact, (1.4) can be generalized in a natural way to the $p$-factorable operators.

THEOREM 1.7. An operator $T \in L(E, F)$ is p-factorable for $p \geqslant 1$ if and only if there are sequences $\left(f_{n}\right)_{n=1}^{\infty} \subset U_{E^{\prime}},\left(y_{n}\right)_{n=1}^{\infty} \subset U_{F}$ and scalars $\left(\lambda_{n}\right)_{n=1}^{\infty}$ with $\sum_{n=1}^{\infty} n^{p}\left|\lambda_{n}\right|$ finite such that $T=\sum_{n=1}^{\infty} \lambda_{n} f_{n} \otimes y_{n}$.

Proof. If $T$ has such a representation then $T=B D A$ where $B, D$ and $A$ are as in (1.4); since $\alpha_{n}(D) \leqslant \Sigma_{k=n+1}^{\infty}\left|\lambda_{k}\right|$ for each $n$ we have 


$$
\sum_{n=1}^{\infty} n^{p-1} \alpha_{n-1}(D) \leqslant \sum_{n=1}^{\infty} n^{p-1} \sum_{k=n}^{\infty}\left|\lambda_{k}\right| \leqslant \sum_{n=1}^{\infty} n^{p}\left|\lambda_{n}\right|
$$

bence $D$ has the desired property.

Conversely, if $T$ factors through a diagonal $D$ having the property that $\sum_{n=1}^{\infty} n^{p-1} \alpha_{n-1}(D)$ converges then $D \sim\left(\lambda_{n}\right)_{n=1}^{\infty}$ where $\left|\lambda_{n+1}\right| \geqslant\left|\lambda_{n}\right|$ for all $n$ (we can assume $\left(\lambda_{n}\right)_{n=1}^{\infty}$ is decreasing by taking a suitable permutation of the indices if necessary). Then

$$
\begin{aligned}
\sum_{n=1}^{\infty} n^{p-1} \alpha_{n-1}(D) & =\sum_{n=1}^{\infty} n^{p-1}\left(\sum_{k=n}^{\infty}\left|\lambda_{k}\right|\right) \\
= & \sum_{n=1}^{\infty}\left(\sum_{i=1}^{n} i^{p-1}\right)\left|\lambda_{n}\right| \geqslant K(p) \sum_{n=1}^{\infty} n^{p}\left|\lambda_{n}\right|
\end{aligned}
$$

where $K(p)$ is a constant depending only on $p$. Let $f_{i}, y_{i}$ and $\mu_{i}$ be as in (1.4). Then $T=\sum_{i=1}^{\infty} \mu_{i} f_{i} \otimes y_{i}$ is a representation of $T$ having the desired property.

Definition 1.8. For $p \geqslant 1$ and $T \in F_{p}(E, F)$ let $f_{p}(T)=$ $\inf \left\{\sum_{n=1}^{\infty} n^{p-1} \alpha_{n-1}(D): D\right.$ is a diagonal from $l^{\infty}$ to $l^{1}$ with $T$ factoring through D).

THEOREM 1.9. For every $p \geqslant 1, F_{p}(E, F)$ is a vector space.

Proof. Let $p \geqslant 1$ and $T_{1}, T_{2} \in F_{p}(E, F)$. Then $T_{i}=V_{i} D_{i} U_{i}$ where $D_{i}: l^{\infty} \rightarrow l^{1}$ is a diagonal with $\Sigma_{n=1}^{\infty} n^{p-1} \alpha_{n-1}\left(D_{i}\right)$ finite for $i=1,2$. Define $I: E \rightarrow E \oplus_{\infty} E$ by $L x=(x, x)$ and $S: F \oplus_{\infty} F \rightarrow F$ by $S(x, y)=x+y$. $I$ and $S$ are bounded linear transformations and we have the commutative diagram

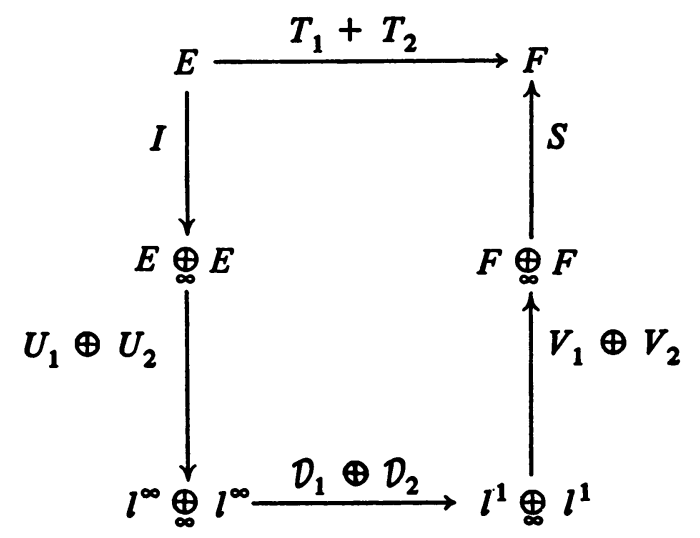

We will first show that $\sum_{n=1}^{\infty} n^{p-1} \alpha_{n-1}\left(D_{1} \oplus D_{2}\right)$ converges. Indeed, if $A_{i}: l^{\infty} \rightarrow l^{1}$ is of rank at most $k$ with $\left\|D_{i}-A_{i}\right\|<\alpha_{k}\left(D_{i}\right)+\epsilon, i=1,2$, then the operator $A=A_{1} \oplus A_{2}: l^{\infty} \oplus_{\infty} l^{\infty} \rightarrow l^{1} \oplus_{\infty} l^{1}$ has rank at most $2 k$. If 


$$
\begin{aligned}
& \xi=\left(\xi^{1}, \xi^{2}\right) \in l^{\infty} \oplus_{\infty} l^{\infty} \text { and }\|\xi\|_{\infty} \leqslant 1 \text { then } \\
& \left\|\left(D_{1} \oplus D_{2}-A\right) \xi\right\|_{\infty}=\left\|\left(D_{1} \xi^{1}-A_{1} \xi^{1}, D_{2} \xi^{2}-A_{2} \xi^{2}\right)\right\|_{\infty} \\
& =\max \left\{\left\|D_{1} \xi^{1}-A_{1} \xi^{1}\right\|_{1},\left\|D_{2} \xi^{2}-A_{2} \xi^{2}\right\|_{1}\right\}<\max _{i=1,2} \alpha_{k}\left(D_{i}\right)+\epsilon .
\end{aligned}
$$

Thus $\alpha_{2 k}\left(D_{1} \oplus D_{2}\right) \leqslant \max _{i=1,2} \alpha_{k}\left(D_{i}\right)$ for each $k$. Since $\alpha_{2 k+1}\left(D_{1} \oplus D_{2}\right) \leqslant$ $\alpha_{2 k}\left(D_{1} \oplus D_{2}\right)$ and $(2 k+1)^{p-1} \leqslant C(p)(2 k)^{p-1}$ for all $k$ it follows that $\Sigma_{k=1}^{\infty} k^{p-1} \alpha_{k-1}\left(D_{1} \oplus D_{2}\right)$ converges if and only if $\Sigma_{k=0}^{\infty}(2 k)^{p-1} \alpha_{2 k}\left(D_{1} \oplus D_{2}\right)$ converges. But

$$
\sum_{k=0}^{\infty}(2 k)^{p-1} \alpha_{2 k}\left(D_{1} \oplus D_{2}\right) \leqslant 2^{p-1} \max _{i=1,2}\left\{\sum_{k=0}^{\infty}(k+1)^{p-1} \alpha_{k}\left(D_{i}\right)\right\} .
$$

We next show that $D_{1} \oplus D_{2}$ factors through a diagonal $D: l^{\infty} \rightarrow l^{1}$ having the property that $\sum_{n=1}^{\infty} n^{p-1} \alpha_{n-1}(D)$ converges. If $(\xi, \eta) \in l^{\infty} \oplus_{\infty} l^{\infty}$ define $R: l^{\infty} \oplus_{\infty} l^{\infty} \rightarrow l^{\infty}$ by $R(\xi, \eta)=\left(\gamma_{i}\right)_{i=1}^{\infty}$ where

$$
\begin{array}{rlrl}
\gamma_{i} & =\xi_{(i+1) / 2} & & \text { if } i \text { is odd, } \\
& =\eta_{i / 2} & \text { if } i \text { is even, }
\end{array}
$$

for $\xi=\left(\xi_{i}\right)_{i=1}^{\infty}$ and $\eta=\left(\eta_{i}\right)_{i=1}^{\infty}$. That is, $R(\xi, \eta)=\left(\xi_{1}, \eta_{1}, \xi_{2}, \eta_{2}, \cdots, \xi_{n}\right.$, $\left.\eta_{n}, \cdots\right) \in l^{\infty}$. Then $R$ is well defined, linear, and $\|R\|=1$. Define $D: l^{\infty} \rightarrow$ $l^{1}$ by $D \sim\left(\delta_{i}\right)_{i=1}^{\infty}$ where

$$
\begin{aligned}
\delta_{i} & =\lambda_{(i+1) / 2} & & \text { if } i \text { is odd, } \\
& =\mu_{i / 2} & & \text { if } i \text { is even, }
\end{aligned}
$$

with $D_{1} \sim\left(\lambda_{i}\right)_{i=1}^{\infty}$ and $D_{2} \sim\left(\mu_{i}\right)_{i=1}^{\infty}$. Now $\|D\| \leqslant \sum_{i=1}^{\infty}\left(\left|\lambda_{i}\right|+\left|\mu_{i}\right|\right)$ so $D \in$ $L\left(l^{\infty}, l^{1}\right)$. Let $F: l^{1} \rightarrow l^{1} \oplus_{\infty} l^{1}$ be defined by $F\left(\xi_{i}\right)_{i=1}^{\infty}=\left(\left(\alpha_{i}\right)_{i=1}^{\infty},\left(\beta_{i}\right)_{i=1}^{\infty}\right)$, $\alpha_{i}=\xi_{2 i-1}$ and $\beta_{i}=\xi_{2 i}$. It follows from the definitions that $D_{1} \oplus D_{2}=F D R$; hence $T_{1}+T_{2}$ factors through the diagonal $D: l^{\infty} \rightarrow l^{1}$. To see that $\sum_{n=1}^{\infty} n^{p-1} \alpha_{n-1}(D)$ is finite we need only observe that $D=B\left(D_{1} \oplus D_{2}\right) A$ where $A: l^{\infty} \rightarrow l^{\infty} \oplus_{\infty} l^{\infty}$ is defined by $A\left(\xi_{i}\right)_{i=1}^{\infty}=\left(\xi^{1}, \xi^{2}\right)$ with $\xi^{1} \doteq\left(\xi_{1}, \xi_{3}, \xi_{5}\right.$, $\left.\cdots, \xi_{2 n+1}, \cdots\right)$ and $\xi^{2}=\left(\xi_{2}, \xi_{4}, \xi_{6}, \cdots, \xi_{2 n}, \cdots\right)$ and $B: l^{1} \oplus_{\infty} l^{1} \rightarrow l^{1}$ is defined by $B\left(\left(\xi_{i}\right)_{i=1}^{\infty},\left(\eta_{i}\right)_{i=1}^{\infty}\right)=\left(\xi_{1}, \eta_{1}, \xi_{2}, \eta_{2}, \cdots\right)$. Now $\|A\|=1$ and $\|B\| \leqslant 2$ so $\alpha_{k}(D) \leqslant\|A\| \alpha_{k}\left(D_{1} \oplus D_{2}\right)\|B\| \leqslant 2 \alpha_{k}\left(D_{1} \oplus D_{2}\right)$.

COROLlary 1.10. For $p \geqslant 1$ and $T_{i} \in F_{p}(E, F), i=1,2$, there is $a$ constant $C(p)$ depending only on $p$ such that $f_{p}\left(T_{1}+T_{2}\right) \leqslant C(p)\left(f_{p}\left(T_{1}\right)+f_{p}\left(T_{2}\right)\right)$. If $p=1$ we can take $C(1)=2$.

We now show that $F_{p}(E, F)$ is complete in the topology generated by $f_{p}$. The following lemma is the first step. 
LEMma 1.11. If $p \geqslant 1$ and $D_{p}\left(l^{\infty}, l^{1}\right)$ denotes the space of all diagonals $D: l^{\infty} \rightarrow l^{1}$ such that $\sigma_{p}(D)=\sum_{n=1}^{\infty} n^{p-1} \alpha_{n-1}(D)$ is finite then $D_{p}(E, F)$ is complete under the topology generated by $\sigma_{p}$.

Proof. Suppose $\left\{D_{n}\right\}_{n=1}^{\infty} \subset D_{p}\left(l^{\infty}, l^{1}\right)$ is $\sigma_{p}$-cauchy. Then there is a diagonal $D: l^{\infty} \rightarrow l^{1}$ such that $\left\|D_{n}-D\right\| \rightarrow 0$. Thus $\alpha_{k}\left(D_{n}-D\right) \rightarrow 0$ uniformly in $k$. Let $M>0$ be such that $\sigma_{p}\left(D_{n}\right) \leqslant M$ for all $n$. Then

$$
\sum_{n=1}^{N} n^{p-1} \alpha_{n-1}(D)=\sum_{n=1}^{N} n^{p-1}\left(\alpha_{n-1}(D)+\alpha_{n-1}\left(D_{m}\right)\right)+\sum_{n=1}^{N} n^{p-1} \alpha_{n-1}\left(D_{m}\right) .
$$

If $m$ is chosen so that $k^{p-1}\left|\alpha_{k-1}(D)-\alpha_{k-1}\left(D_{m}\right)\right|<1 / N$ for all $k, 1 \leqslant k \leqslant N$, then $\sum_{n=1}^{N} n^{p-1} \alpha_{n-1}(D)<M+1$. That is, $\sigma_{p}(D)$ is finite. That $\sigma_{p}\left(D-D_{m}\right)$ $\longrightarrow 0$ is clear.

THEOREM 1.12. For each $p \geqslant 1, F_{p}(E, F)$ is complete under the topology generated by $f_{p}$.

Proof. If $\left(T_{n}\right)_{n=1}^{\infty} \subset F_{p}(E, F)$ is $f_{p}$-cauchy then there is $T \in L(E, F)$ such that $\left\|T_{n}-T\right\| \rightarrow 0$. Choose a subsequence $\left(T_{n_{i}}\right)_{i=1}^{\infty}$ of $\left(T_{n}\right)_{n=1}^{\infty}$ such that $f_{p}\left(T_{n_{i+1}}-T_{n_{i}}\right)<2^{-8 i}$ for each $i$ and let $S_{i}=T_{n_{i+1}}-T_{n_{i}}$. Then $T=$ $T_{n_{1}}+\sum_{i=1}^{\infty} S_{i}=\sum_{i=0}^{\infty} S_{i}$ where $S_{0}=T_{n_{1}}$. Write $S_{i}=V_{i} D_{i} U_{i}$ with $\left\|V_{i}\right\|=$ $\left\|U_{i}\right\|=1$ and $\sum_{n=1}^{\infty} n^{p-1} \alpha_{n-1}\left(D_{i}\right) \leqslant f_{p}\left(S_{i}\right)+2^{-8 i}$. Then we can assume $\left\|V_{i}\right\|=$ $\left\|U_{i}\right\|=2^{-i}$ for each $i \geqslant 1$ and $\sum_{n=1}^{\infty} n^{p-1} \alpha_{n-1}\left(D_{i}\right)<2^{-5 i}$ and $\left\|V_{0}\right\|=$ $\left\|U_{0}\right\|=1$ with $\sum_{n=1}^{\infty} n^{p-1} \alpha_{n-1}\left(D_{0}\right)<f_{p}\left(S_{0}\right)+2^{-6 i}$. Then $\Sigma_{n=0}^{\infty}\left\|V_{n}\right\|$ and $\Sigma_{n=0}^{\infty}\left\|U_{n}\right\|$ converge so there are $U \in L\left(E, l^{\infty}\right)$ and $V \in L\left(l^{1}, F\right)$ such that $U=\Sigma_{n=0}^{\infty} U_{n}$ and $V=\Sigma_{n=0}^{\infty} V_{n}$. Also $\sum_{n=0}^{\infty} \sigma_{p}\left(D_{n}\right)$ converges and so by (1.11) there is $D \in D_{p}\left(l^{\infty}, l^{1}\right)$ with $D=\sum_{n=0}^{\infty} D_{n}$. Now

$$
\begin{aligned}
\left\|V D U-\sum_{i=0}^{n} V_{i} D_{i} U_{i}\right\| & \leqslant\left\|\sum_{i=n+1}^{\infty} V_{i} P_{i}\right\|\left\|\sum_{i=n+1}^{\infty} D_{i} U_{i}\right\| \\
& \leqslant\left\|\sum_{i=n+1}^{\infty} V_{i} P_{i}\right\|\left\|\sum_{i=n+1}^{\infty} D_{i} Q_{i}\right\|\left\|\sum_{i=n+1}^{\infty} U_{i}\right\| \\
& \leqslant \sum_{i=n+1}^{\infty}\left\|V_{i}\right\| \sum_{i=n+1}^{\infty}\left\|D_{i}\right\| \sum_{i=n+1}^{\infty}\left\|U_{i}\right\| \underset{n}{\rightarrow} 0
\end{aligned}
$$

where $P_{i}:\left(\sum_{i=1}^{\infty} \bigoplus l_{i}^{1}\right) \rightarrow l_{i}^{1}$ and $Q_{i}:\left(\sum_{i=1}^{\infty} \bigoplus l_{i}^{\infty}\right) \rightarrow l_{i}^{\infty}$ are the natural projections (viewing $l^{1}$ and $l^{\infty}$ as $\left(\Sigma_{i=1}^{\infty} \oplus l_{i}^{1}\right)$ and $\left(\Sigma_{i=1}^{\infty} \oplus l_{i}^{\infty}\right)$ respectively). Thus $V D U=\sum_{i=0}^{\infty} S_{i}=T$.

2. Relationships between various classes of operators. In this section we 
study the relationship between the spaces of operators $F_{p}(E, F), F_{p}(E, F)$, $L^{(p)}(E, F)$ and $l^{p}(E, F)$. We obtain several factorization theorems pertaining to operators of these various types.

It is clear from the definitions that $F_{p}(E, F) \subset F_{1 / p}(E, F)$ for each $p \geqslant 1$ and $f_{p}(T) \geqslant F_{1 / p}(T)$. It also follows immediately that for $0<p \leqslant 1 / 2, F_{p}(E, F)$ $\subset F_{q}(E, F)$ for every $q, 1 \leqslant q<(1-p) / p$, and $f_{q}(T) \leqslant K(p) F_{p}(T)$ where $K(p)$ is a constant depending only on $p$. In fact, this is the best result possible. To see this let $T: l^{1} \rightarrow l^{1}$ be the diagonal $T \sim\left(\lambda_{n}\right)_{n=1}^{\infty}$ where $\lambda_{n}=$ $n^{-1 / p}[\ln (n+1)]^{-1}$ for $0<p \leqslant 1$. Then $n^{1 / p} \lambda_{n} \rightarrow 0$; hence $T \in F_{p}\left(l^{1}, l^{1}\right)$. If $T \in F_{m}\left(l^{1}, l^{1}\right), m=(1-p) / p$, then $T=A D B$ where $D: l^{\infty} \rightarrow l^{1}$ is a diagonal with the property that $\sum_{n=1}^{\infty} n^{m-1} \alpha_{n-1}(D)$ converges. In particular, since $\alpha_{n}(T) \leqslant\|A\|\|B\| \alpha_{n}(D), \sum_{n=1}^{\infty} n^{m-1} \alpha_{n-1}(T)$ converges. But $\alpha_{n}(T)=\lambda_{n+1}[6]$, [7] for each $n$. This contradiction shows that $T \notin F_{m}\left(l^{1}, l^{1}\right)$ where $m=$ $(1-p) / p$.

Pietsch [6], [7] has shown that every $T \in l^{p}(E, F), 0<p \leqslant 1$, has a rep- - . resentation $T=\sum_{i=1}^{\infty} \lambda_{i} f_{i} \otimes y_{i}$ where $\left(f_{i}\right)_{i=1}^{\infty} \subset U_{E^{\prime}},\left(y_{i}\right)_{i=1}^{\infty} \subset U_{F}$ and $\left(\lambda_{i}\right)_{i=1}^{\infty} \in l^{p}$; that is $l^{p}(E, F) \subset L^{(p)}(E, F)$ for each $p, 0<p \leqslant 1$. We now show that, in general, the opposite inclusion does not hold.

Proposition 2.1. Let $0<q \leqslant 1$ and $T \in L^{(q)}(E, F)$. Then $T$ factors through a diagonal from $l^{\infty}$ to $l^{1}$ which is of type $l^{p}, 1 / p=1 / q-1$. In particular, $T$ is of type $l^{p}, 1 / p=1 / q-1$, and this is the best result possible. If $0<q \leqslant 2 / 3$ then $T_{a}$ is of type $l^{p}$ where $1 / p=1 / q-3 / 2$.

Proof. If $T \in L^{(q)}(E, F)$ then $T=\sum_{i=1}^{\infty} \lambda_{i} f_{i} \otimes y_{i}$ where $\left\|f_{i}\right\|_{,}\left\|y_{i}\right\| \leqslant 1$, $\left|\lambda_{i}\right| \geqslant\left|\lambda_{i+1}\right|$ for all $i$, and $\left(\lambda_{i}\right)_{i=1}^{\infty} \in l^{q}$. Thus $T$ has the factorization

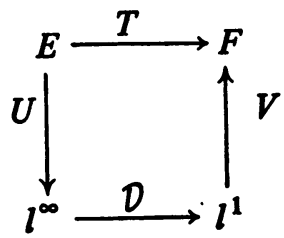

where $U x=\left(\left\langle x, f_{i}\right\rangle\right)_{i=1}^{\infty}, D \sim\left(\lambda_{i}\right)_{i=1}^{\infty}$, and $V=\Sigma_{i=1}^{\infty} e_{i} \otimes y_{i}$. Now $D=D_{2} D_{1}$ where

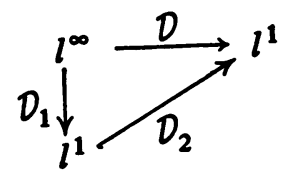

with $D_{1} \sim\left(\lambda_{i}^{q}\right)_{i=1}^{\infty}$ and $D_{2} \sim\left(\lambda_{i}^{1-q}\right)_{i=1}^{\infty}$. Since $\alpha_{k}\left(D_{2}\right)=\left|\lambda_{k+1}\right|^{1-q}[6], D_{2}$ 
is of type $l^{q /(1-q)}$; hence $T=V D_{2} D_{1} U$ is also of type $l^{q /(1-q)}$.

If $q \leqslant 2 / 3$, write $D=D_{3} D_{2} D_{1}$ where

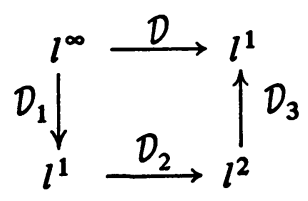

with $D_{3} \sim\left(\lambda_{i}^{q / 2}\right)_{i=1}^{\infty}, D_{2} \sim\left(\lambda_{i}^{1-3 q / 2}\right)_{i=1}^{\infty}$ and $D_{1} \sim\left(\lambda_{i}^{q}\right)_{i=1}^{\infty}$. Since $\alpha_{k}\left(D_{2}\right) \leqslant$ $\left|\lambda_{k+1}\right|^{1-3 q / 2}$ it follows that $D_{2}$ is of type $l^{p}, 1 / p=1 / q-3 / 2$. To finish the proof observe that $T_{a}=V D_{3} P D_{2} D_{1} U$ where $P: l^{2} \rightarrow \overline{\left(V D_{3}\right)^{-1}(T(E))}$ is the orthogonal projection.

We now show that the result $T \in L^{(q)}(E, F), 0<q<1$, implies that $T \in$ $l^{p}(E, F), 1 / p=1 / q-1$, is the best result possible. Indeed, for $0<q<1$ we will construct a diagonal $T: l^{\infty} \rightarrow l^{1}$ which is strongly $q$-summable but not of type $l^{[q /(1-q)]-\epsilon}$ for any $\epsilon>0$. For fixed $q, 0<q<1$, choose $\beta$ such that $\beta q>1$. Let $\beta_{n}=(n+1)^{(q-1) / q}[\ln (n+1)]^{-\beta}$ and define $T: l^{\infty} \rightarrow l^{1}$, $T \sim\left(\lambda_{n}\right)_{n=1}^{\infty}$, where $\lambda_{n}=\beta_{n}-\beta_{n+1}$. It follows from the mean value theorem and the choice of $\beta$ that $\Sigma_{n=1}^{\infty} \mid \beta_{n}-\beta_{n+1}{ }^{q}$ is finite; hence $T \in$ $L^{(q)}\left(l^{\infty}, l^{1}\right)$. Since $\alpha_{n}(T)=\sum_{i=n+1}^{\infty} \lambda_{i}=\beta_{n+1}[2], T$ is not of type $l^{[q /(1-q)]-\epsilon}$ by the choice of $\beta_{n}$ and $\beta$.

We point out that in [4] Markus proved that $L^{(p)}(E, F) \subset l^{p /(1-p)}(E, F)$ (using completely different techniques) and remarked without proof that $p /(1-p)$ was the best result possible.

The following proposition is well known and easy to prove'.

PROPOSITION 2.2. If $\left(\lambda_{n}\right)_{n=1}^{\infty}$ is any sequence of positive scalars such that $\sum_{n=1}^{\infty} n^{p} \lambda_{n}$ converges for $p \geqslant 1$ then $\left(\lambda_{n}\right)_{n=1}^{\infty} \in l^{(1+\epsilon) /(p+1)}$ for every $\epsilon>0$ and this is the best result possible. If $\left(\lambda_{n}\right)_{n=1}^{\infty} \in l^{1 /(p+1)}$ for $p \geqslant 1$ then $\sum_{n=1}^{\infty} n^{p} \lambda_{n}$ converges and this is the best result possible.

Proposition 2.3. If $p>1$ and $T \in F_{p}(E, F)$ then $T$ factors through $a$ diagonal $D: l^{1} \rightarrow l^{2}$ of type $l^{1 /(p-1)}$. In particular $T_{a}$ is of type $l^{1 /(p-1)}$. Moreover $T$ factors through a diagonal $D_{0}: l^{1} \rightarrow l^{1}$ of type $l^{2 /(2 p-1)}$; hence $T \in l^{2 /(2 p-1)}(E, F)$.

Proof. If $T \in F_{p}(E, F)$ then, by (1.7), $T=\Sigma_{n=1}^{\infty} \lambda_{n} f_{n} \otimes y_{n}$ where $\left\|f_{n}\right\|$, $\left\|y_{n}\right\| \leqslant 1$ and $\sum_{n=1}^{\infty} n^{p}\left|\lambda_{n}\right|$ converges. In particular, $\left(\lambda_{n}\right)_{n=1}^{\infty} \in l^{2 /(2 p+1)}$ by (2.2). Therefore we can factor $T$

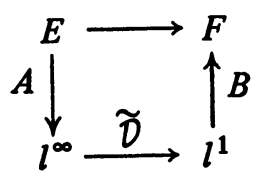


where $A, B$ and $\tilde{D}$ are as in (1.4). Now we can write $\widetilde{D}=D_{2} D D_{3}$ where

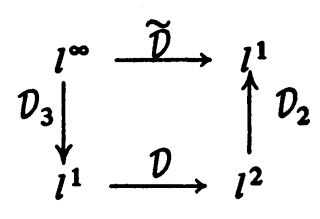

with $D_{3} \sim\left(\lambda_{n}^{2 /(2 p+1)}\right)_{n=1}^{\infty}, D \sim\left(\lambda_{n}^{2(p-1) /(2 p+1)}\right)_{n=1}^{\infty}$ and $D_{2} \sim\left(\lambda_{n}^{1 /(2 p+1)}\right)_{n=1}^{\infty}$ Thus $\alpha_{k}(D) \leqslant\left|\lambda_{k+1}\right|^{2(p-1) /(2 p+1)}$ and so $D \in l^{1 /(p-1)}\left(l^{1}, l^{2}\right)$; it follows that $T_{a} \in l^{1 /(p-1)}\left(E, \frac{T(E))}{T}\right.$. If we let $D_{0}=D_{2} D$ then $D_{0} \sim\left(\lambda_{n}^{(2 p-1) /(2 p+1)}\right)_{n=1}^{\infty}$. Since $\alpha_{k}\left(D_{0}\right) \leqslant\left|\lambda_{k+1}\right|^{(2 p-1) /(2 p+1)}$ we have the desired result.

If $p=1$ we have the following version of (2.3).

Proposition 2.4. If $T \in F_{1}(E, F)$ then $T_{a}$ is of type $l^{2}$. Indeed, $T$ factors through a diagonal from $l^{\infty}$ to $l^{2}$ which is of type $l^{2}$.

Proof. For $T \in F_{1}(E, F)$ choose a representation $T=\sum_{n=1}^{\infty} \lambda_{n} f_{n} \otimes y_{n}$ with $\left\|f_{n}\right\|,\left\|y_{n}\right\| \leqslant 1$ and $\sum_{n=1}^{\infty} n\left|\lambda_{n}\right|$ finite. Then $T=V D U$ where

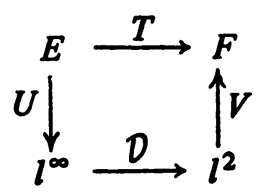

with $U x=\left(\left\langle x, f_{n}\right\rangle\right)_{n=1}^{\infty}, D \sim\left(\lambda_{n}^{2 / 3}\right)_{n=1}^{\infty}$, and $V=\sum_{n=1}^{\infty} \lambda_{n}^{1 / 3} e_{n} \otimes y_{n}$. The operators $U, V$ and $D$ are well defined by (2.2). Now $D=B A$ where

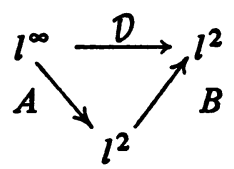

and $A \sim\left(\lambda_{n}^{1 / 3}\right)_{n=1}^{\infty}$ and $B \sim\left(\lambda_{n}^{1 / 3}\right)_{n=1}^{\infty}$. Since $B$ is of type $l^{2}[6], D$ is also of type $l^{2}$ and the result follows.

We point out that there are operators whose astrictions are of type $l^{2}$ yet the operators are not 1-factorable. Indeed, choose any Hilbert-Schmidt operator on Hilbert space which is not of type $l^{1}$. Then its astriction is also HilbertSchmidt, hence of type $l^{2}$ [6], but the operator is certainly not 1 -factorable.

We next consider the relationship between $p$-factorable and strongly $p$-summable operators.

Proposition 2.5. Let $p \geqslant 1$ and $T \in L^{(q)}(E, F)$ where $q=1 /(p+1)$. Then $T$ is p-factorable. In particular, if $T$ is of type $l^{q}, q=1 /(p+1)$, then 
$T$ is p-factorable and these are the best results possible.

The proof is immediate from (2.2).

It follows from (1.6) that for $p$-factorable operators $T, p \geqslant 1$, $\sum_{n=1}^{\infty} n^{p-1} \alpha_{n-1}(T)$ converges; hence, by (2.2), $T$ is of type $l^{q}$ for every $q>1 / p$. We next show that these are the best results possible.

Proposition 2.6. If $p \geqslant 1$ and $T$ is p-factorable then $\sum_{n=1}^{\infty} n^{p-1} \alpha_{n-1}(T)$ converges and this is the best result possible. Moreover, $T$ is of type $l^{(1 / p)+\epsilon}$ for every $\epsilon>0$ and, for $p>1$, this is the best result possible.

PROof. The first part of the proposition follows from the definition of the $p$-factorable operators and the fact that for $T=B D A, \alpha_{k}(T) \leqslant\|A\|\|B\| \alpha_{k}(D)$.

If $p>1$ let $\beta_{n}=n^{-p} \ln ^{-2}(n+1)$ and $\lambda_{n}=\beta_{n}-\beta_{n+1}$. Define $T$ : $l^{\infty} \rightarrow l^{1}$ by $T \sim\left(\lambda_{n}\right)_{n=1}^{\infty}$. It follows from the mean value thecrem that $\sum_{n=1}^{\infty} n^{p}\left|\lambda_{n}\right|$ converges. Since $\alpha_{n}(T)=\sum_{k=n+1}^{\infty}\left|\lambda_{k}\right|$ we have $\sum_{n=1}^{\infty} n^{p-1} \alpha_{n-1}(T)$ finite. But

$\alpha_{n}(T)=\sum_{k=n+1}^{\infty}\left|\lambda_{k}\right|=\beta_{n+1}$ and $\sum_{n=1}^{\infty} n^{(p-1)+\epsilon} \alpha_{n-1}(T)=\sum_{n=1}^{\infty} \frac{n^{(p-1)+\epsilon}}{(n+1)^{p} \ln ^{2}(n+1)}$

which diverges for any $\epsilon>0$.

Now let $p>1$ and $T \in F_{p}(E, F)$. It follows from the above and (2.2) that $T$ is of type $l^{(1 / p)+\epsilon}$ for every $\epsilon>0$. To see that this is the best result possible let $\beta_{n}=n^{-p} \ln ^{-p}(n+1), \lambda_{n}=\beta_{n}-\beta_{n+1}$ and define $T: l^{\infty} \rightarrow l^{1}$ by $T \sim\left(\lambda_{n}\right)_{n=1}^{\infty}$. It again follows that $\sum_{n=1}^{\infty} n^{p} \lambda_{n}$ converges; hence $T \in F_{p}\left(l^{1}, l^{\infty}\right)$. But $\alpha_{k}(T)=\sum_{n=k+1}^{\infty} \lambda_{n}=\beta_{k+1}$ and $\left(\beta_{k}\right)_{k=1}^{\infty} \notin l^{1 / p}$; hence $T$ is not of type $l^{1 / p}$.

We point out that for $p=1$ the result $F_{p}(E, F) \subset l^{(1+\epsilon) / p}(E, F)$ is not the best possible. Indeed, the first part of (2.6) gives $F_{1}(E, F) \subset l^{1}(E, F)$.

As a partial converse to (2.6) we have the following proposition.

Proposition 2.7. Let $p \geqslant 1$ and $T \in L(E, F)$. If $\sum_{n=1}^{\infty} n^{p} \alpha_{n-1}(T)$ converges then there are sequences $\left(f_{n}\right)_{n=1}^{\infty} \subset U_{E^{\prime}},\left(y_{n}\right)_{n=1}^{\infty} \subset U_{F}$ and scalars $\left(\lambda_{n}\right)_{n=1}^{\infty}$ with $\sum_{n=1}^{\infty} n^{q}\left|\lambda_{n}\right|$ finite for every $q, 0<q<p$, such that $T=$ $\sum_{n=1}^{\infty} \lambda_{n} f_{n} \otimes y_{n}$.

Proof. Since $\sum_{n=1}^{\infty} n^{p} \alpha_{n-1}(T)$ converges, $\left(\alpha_{n}(T)\right)_{n=0}^{\infty} \in l^{r}$ for every $r>$ $1 /(p+1)$ by (2.2); in particular $T \in l^{r}(E, F)$ for $1 /(p+1)<r \leqslant 1$ and so by [6] there are sequences $\left(f_{n}\right)_{n=1}^{\infty} \subset U_{E^{\prime}},\left(y_{n}\right)_{n=1}^{\infty} \subset U_{F}$ and a nonincreasing sequence of scalars $\left(\lambda_{n}\right)_{n=1}^{\infty} \in l^{r}$ such that $T=\sum_{n=1}^{\infty} \lambda_{n} f_{n} \otimes y_{n}$. Now 
$\left(n^{1 / r}\left|\lambda_{n}\right|\right)_{n=1}^{\infty}$ is bounded; hence $\sum_{n=1}^{\infty} n^{1 / r}\left|\lambda_{n}\right| n^{-(1+\epsilon)}=\sum_{n=1}^{\infty} n^{\dot{p}-\delta}\left|\lambda_{n}\right|$ converges for $\delta=\delta(\epsilon, r)>0$.

If $T \in F_{p}(E, F), S_{1} \in L(G, E)$ and $S_{2} \in L(F, X)$ then $T S_{1}$ and $S_{2} T$ are $p$-factorable. For $p$-factorable $T$ and $q$-factorable $S, p, q \geqslant 1$, we have the following composition formula.

PROPOSITION 2.8. For $p, q, S$ and $T$ as above, the composition $S T$ is $r$ factorable for every $r, 1 \leqslant r<p+q-1$.

Proof. We can write $T=\sum_{n=1}^{\infty} \lambda_{n} f_{n} \otimes y_{n}$ with $\left\|f_{n}\right\|,\left\|y_{n}\right\| \leqslant 1$ and $\sum_{n=1}^{\infty} n^{p}\left|\lambda_{n}\right|$ finite. The operator $S$ also has a representation $S=\sum_{n=1}^{\infty} \mu_{n} g_{n} \otimes x_{n}$ with $\left\|g_{n}\right\|,\left\|x_{n}\right\| \leqslant 1$ and $\sum_{n=1}^{\infty} n^{q}\left|\mu_{n}\right|$ finite. Since $\alpha_{n}(S) \leqslant \sum_{i=n+1}^{\infty}\left|\mu_{i}\right|$ we have

$$
n^{q} \alpha_{n-1}(S) \leqslant n^{q} \sum_{i=n}^{\infty}\left|\mu_{i}\right| \leqslant \sum_{i=n}^{\infty} i^{q}\left|\mu_{i}\right|
$$

thus $n^{q} \alpha_{n-1}(S) \rightarrow 0$. By (2.6), $\sum_{n=1}^{\infty} n^{p-1} \alpha_{n-1}(T)$ converges; hence

$$
\begin{aligned}
\sum_{n=1}^{\infty} n^{p+q-1} \alpha_{n-1}(S T) & \leqslant \sum_{n=1}^{\infty}(2 n)^{p+q-1} \alpha_{2 n}(S T)+\sum_{n=1}^{\infty}(2 n+1)^{p+q-1} \alpha_{2 n+1}(S T) \\
& \leqslant K(p, q) \sum_{n=1}^{\infty} n^{p+q-1} \alpha_{2 n}(S T) \\
& \leqslant K(p, q) \sum_{n=1}^{\infty}\left(n^{p-1} \alpha_{n-1}(T)\right)\left(n^{q} \alpha_{n-1}(S)\right)<\infty
\end{aligned}
$$

By (2.7), $S T$ has a representation $S T=\sum_{n=1}^{\infty} \beta_{n} h_{n} \otimes z_{n}$ where $\left\|h_{n}\right\|,\left\|z_{n}\right\| \leqslant 1$ and $\sum_{n=1}^{\infty} n^{r}\left|\beta_{n}\right|$ converges for every $r, 1 \leqslant r<p+q-1$. The result now follows from (1.7).

We now give a summary of this section.

Theorem 2.9 (Summary). (i) If $0<p<1$ then

$$
L^{(p)}(E, F) \subset l^{p /(1-p)}(E, F)
$$

and this is the best result possible.

(ii) For $0<p \leqslant 1, l^{p}(E, F) \subset L^{(p)}(E, F)$.

(iii) For $p \geqslant 1, L^{(q)}(E, F) \subset F_{p}(E, F), q=1 /(p+1)$. In particular, $l^{q}(E, F) \subset F_{p}(E, F)$.

(iv) For $p \geqslant 1, F_{p}(E, F) \subset L^{(r)}(E, F)$ for every $r, 1 \geqslant r>1 /(p+1)$ and this is the best result possible. 
(v) For $p>1, F_{p}(E, F) \subset l^{q}(E, F)$ for every $q>1 / p$ and this is the best result possible. If $p=1$ we have $F_{1}(E, F) \subset l^{1}(E, F)$ and this is the best result possible.

(vi) For $p \geqslant 1, F_{p}(E, F) \subset F_{1 / p}(E, F)$.

(vii) For $0<p \leqslant 1 / 2, F_{p}(E, F) \subset F_{q}(E, F)$ for every $q, 1 \leqslant q<(1-p) / p$. and this is the best result possible.

(viii) If $0<q \leqslant 1 / 2$ then $L^{(q)}(E, F) \subset F_{r}(E, F)$ for $r=q /(1-q)$. In particular, $l^{q}(E, F) \subset F_{r}(E, F)$.

(ix) For $0<p \leqslant 1 / 2, F_{p}(E, F) \subset L^{(r)}(E, F)$ for every $r, p /(1-p)<r \leqslant 1$. If we let $l_{R}^{p}(E, F)$ denote the collection of operators from $E$ to $F$ whose astrictions are of type $l^{p}$ then (2.3) and (2.4) give the following result.

(x) For $p>1, F_{p}(E, F) \subset l_{R}^{1 /(p-1)}(E, F)$ and for $p=1, F_{1}(E, F) \subset$ $l_{R}^{2}(E, F)$.

We feel a few words about the class of operators $l_{R}^{p}(E, F)$ are in order. It is not difficult to give examples of operators $T$ and spaces $E, F$ for which $\alpha_{k}(T) \neq \alpha_{k}\left(T_{a}\right)$. For example, if $T: l^{1} \rightarrow l^{\infty}$ is natural injection then $\alpha_{k}(T)=$ $1 / 2$ and $\alpha_{k}\left(T_{a}\right)=1$ for all $k$ [2]. It is not known if the astriction of an operator of type $l^{p}$ is again of type $l^{p}$. We suspect this is not the case; however, we have been unable to construct such an example, even for $p=1$. Results of Retherford and Stegall [9] on fully nuclear operators and of Grothendieck [1, $\S 2]$ on the astrictions of strongly $p$-summable operators support our conjecture. We have shown [3] that the astriction of an operator of type $l^{2 / 3}$ is nuclear (hence such an operator is fully nuclear [9]) and that the astriction of an operator of type $l^{p}, 0<p \leqslant 1$, is of type $l^{r}, 1 / r=1 / p-1$.

\section{BIBLIOGRAPHY}

1. A. Grothendieck, Produits tensoriels topologiques et espaces nucléaires, Mem. Amer. Math. Soc. No. 16, 1955. MR 17, 763.

2. C. V. Hutton, J. S. Morrell and J. R. Retherford, Approximation numbers and Kolmogoroff diameters of bounded linear operators, Bull. Amer. Math. Soc. 80 (1974), 462-466.

3. C. V. Hutton, $t^{p}(E, F)$ and sets of type $\imath^{p}$, Math. Ann. (submitted).

4. A. S. Markus, Certain criteria for the completeness of a system of root-vectors of a linear operator in a Banach space, Mat. Sb. 70 (112) (1966), 526-561; English transl., Amer. Math. Soc. Transl. (2) 85 (1969), 51-91. MR 35 \#7151.

5. B. S. Mitjagin and A Pekczyński, Nuclear operators and approximative dimension, Proc. Internat. Congr. Math., Moscow, 1966, Izdat. "Mir", Moscow, 1968; English transl., Amer. Math. Soc. Transl. (2) 70 (1968), 137-145. MR 39 \#6046.

6. A. Pietsch, Nukleare lokalconvexe Räume, Academie-Verlag, Berlin, 1965. MR 31 \#6114. 
7. A. Pietsch, Einige neue Klassen von kompakten linearen Abbildungen, Rev. Math. Pures Appl. (Bucharest) 8 (1963), 427-447. MR 31 \#3874.

8. - s-numbers of operators on Banach spaces, Studia Math. (to appear).

9. J. R. Retherford and C. P. Stegall, Fully nuclear and completely nuclear operators with applications to $L_{1}$ and $L_{\infty}$ spaces, Trans. Amer. Math. Soc. 163 (1972), 457-492.

10. H. Weyl, Inequalities between the two kinds of eigenvalues of a linear transformation, Proc. Nat. Acad. Sci. U. S. A. 35 (1949), 408-411. MR 11, 37.

DEPARTMENT OF MATHEMATICS, VIRGINIA POLYTECHNIC INSTITUTE AND STATE UNIVERSITY, BLACKSBURG, VIRGINIA 24061

Current address: Department of Mathematics, The Catholic University of America, Washington, D. C. 20064 